\title{
Conductas no deseadas en equinos
}

\author{
Undesired behaviours in horses \\ TA Tadich ${ }^{a b *}$, O Araya ${ }^{c}$ \\ aBecario CONICYT, Programa Doctorado en Ciencias Veterinarias, Facultad de Ciencias Veterinarias, \\ Universidad Austral de Chile, Valdivia, Chile. \\ 'Instituto de Ciencia Animal, Facultad de Ciencias Veterinarias, Universidad Austral de Chile, Valdivia, Chile. \\ 'Instituto de Ciencias Clínicas, Facultad de Ciencias Veterinarias, Universidad Austral de Chile, Valdivia, Chile.
}

\begin{abstract}
SUMMARY
Animal welfare has become a relevant ongoing area of research and a great number of studies can be found in the literature assessing the welfare of different animal species by the use of direct or indirect methods. Within the direct methods, behavioural parameters are acquiring more importance, in particular those related with the performance of abnormal behaviours. This because of the physical, physiological and mental consequences that these behaviours carry with them. There are numerous publications providing information on the diverse abnormal behavioural displays shown by equines, many times these make their understanding and classification difficult and confusing. The present review aims to describe those behaviours considered abnormal or undesirable in horses emphasising those considered as stereotypes. For this purpose they will be classified providing also information about their possible causes, form of presentation, function and prevention. On the other hand implications of these behaviours on the horse's welfare will be briefly explained.
\end{abstract}

Palabras clave: conducta anormal, estereotipia, equino, bienestar. Key words: abnormal behaviour, stereotype, equine, welfare.

\section{INTRODUCCIÓN}

Los equinos (Equus caballus) presentan un repertorio conductual característico para su especie, entendiéndose por conducta las acciones y reacciones de un organismo frente a su medio ambiente con una función determinada. Estas acciones y reacciones se encuentran controladas de manera interna por el sistema endocrino y neurológico, y externamente por el medio ambiente (Duncan 1998). Debido a que los equinos en estado salvaje (por ejemplo, Equus przewalskii) se extinguieron antes de que se estudiara su comportamiento, la información que se maneja actualmente respecto a su organización social y conductual proviene de estudios realizados durante los últimos 20 años en poblaciones de equinos que se devolvieron a su estado salvaje luego de muchas generaciones en cautiverio (Keiper 1986).

En el equino se ha descrito una serie de conductas consideradas anormales, entendiéndose como tales aquellas que presentan una desviación significativa del promedio y que no presentan una función obvia o pueden infringir un daño sobre el mismo animal (Mason 1991). Algunas

Aceptado: 28.10.2009.

* Programa de Doctorado en Ciencias Veterinarias, Escuela de Graduados, Facultad de Ciencias Veterinarias, UACh, Casilla 567, Valdivia, Chile; tamaratadich@uach.cl conductas son inadecuadamente catalogadas como "anormales", debido a una falta de conocimiento del repertorio de conductas normales que presenta el animal o porque representan una molestia para el propietario. Según Broom y Fraser $\left(2007^{\mathrm{a}}\right)$, las anormalidades tienden a ocurrir cuando la frecuencia de los movimientos, intensidad de las acciones o contexto en el cual ocurre la conducta son diferentes a lo normal. En ciertas ocasiones estas conductas no deseadas parecen tener una función adaptativa para el animal, permitiéndole de esta manera enfrentar el cautiverio (Boyd 1986). En términos generales las conductas no deseadas tienden a reducir el rendimiento deportivo, el valor del animal o ambos (Fraser 1992), debido a la posibilidad de predisponer a ciertas patologías específicas (Mills y col 2005). Por lo tanto, ellas debieran tratar de prevenirse en la medida que sea posible, ya que una vez que se instalan en el repertorio conductual del animal son muy difíciles de revertir (Boyd 1986).

La mayoría de las conductas no deseadas en los equinos resultan de dos cambios fundamentales que caracterizan su vida en estado salvaje, los que se encuentran ausentes en cautiverio. Por una parte, la estructura social se ve alterada, ya que el equino es un animal gregario que forma parte de un harem o de bandas de machos juveniles, y que raramente se mantiene solitario por opción propia, lo cual generalmente no ocurre dentro del manejo en cautiverio. Por otra parte, la disponibilidad ilimitada de tiempo para 
forrajear se ve notablemente restringida en cautiverio (Boyd 1986, Houpt 1986). Un estudio realizado entre 1995 y 1999 sobre una población de 225 equinos demostró que un $34 \%$ de la población presentaba algún tipo de conducta anormal (Waters y col 2002).

Los problemas conductuales pueden clasificarse según su etiología (carencias nutricionales, sociales, ambientales, de origen fisiológico o médico) o según la forma en que se manifiestan (orales-ingestivos, somáticos, reactivos) (Houpt 1986, Fraser 1992). Broom y Fraser (2007ª) las clasifican en estereotipadas y otras conductas anormales, según a quien van dirigidas (a sí mismo, a otros individuos o a algún objeto). Otra forma de clasificarlos es en conductas vacías, redirigidas, desplazadas, aprendidas y estereotipadas (McGreevy 2004). Esta última clasificación parece ser más clara, permitiendo además en cada caso establecer su origen y forma de presentación.

Además de saber cómo clasificar estas conductas no deseadas, es importante comprender si ellas son el signo de sufrimiento mental, de un manejo cruel, estrés o evidencia de un manejo y ambiente inadecuado por parte de las personas (Fraser 1992). Por esta razón, es necesario entender las causas, función y forma de prevenir estas conductas. Generalmente ellas han sido asociadas con aburrimiento dentro de la pesebrera, considerándose que estas conductas, en alguna medida, permitirían al animal aliviar el estrés, siendo ellas un indicador confiable de la falta de bienestar en el animal (Kiley-Worthington 1977).

\section{IMPLICACIONES DE LAS CONDUCTAS NO DESEADAS SOBRE EL BIENESTAR EQUINO}

Broom (1986) define el bienestar animal como "el estado de un animal en relación a sus intentos por adaptarse al medio ambiente": El animal puede adaptarse de manera exitosa, necesitar de un mayor esfuerzo para adaptarse o simplemente fallar en sus intentos de adaptación, lo cual puede incluso llevar a la muerte del individuo. La definición de bienestar animal tiene varias implicancias, entre ellas el ser una característica inherente al animal y no algo que se le entrega, el poder variar de muy bueno a muy pobre y poder ser medible de manera científica e independiente a consideraciones de tipo moral (Broom 1991).

El concepto propuesto por Broom resalta los aspectos científicos del bienestar animal, describiéndolo como una característica medible; sin embargo, el tema ético (cómo debiéramos tratar a los animales) y legal (cómo debemos tratar a los animales) no son aspectos menores a considerar, ya que estos van a afectar la forma en que el tema se trate de acuerdo a la idiosincrasia de cada país. Estas diferencias pueden llevar a interpretaciones distintas del concepto de bienestar en diferentes países, estableciendo cada uno parámetros o estándares de bienestar diferentes (Fraser 2003). También es importante considerar que en muchos países no desarrollados o en vías de desarrollo aún existen problemas de bienestar humano, haciendo difícil y en ocasiones imposible exigir el cumplimiento de estándares de bienestar animal.

A través de una perspectiva un poco diferente Duncan y Fraser (1997) consideran el concepto de bienestar animal basado en tres aspectos fundamentales: 1) las experiencias del animal, tales como placer o dolor; 2) el funcionamiento biológico normal del individuo, y 3 ) la naturalidad de la especie (telos), asegurando que el animal pueda realizar el repertorio conductual completo de su especie. Esta definición parece ser más completa, ya que toma en cuenta los aspectos físicos, mentales y conductuales del individuo; además, se encuentra en armonía con la definición propuesta por Dawkins (2008), quien establece que se debiera poner énfasis en un estado de salud adecuado y que el animal pueda obtener lo que quiere, entregando así una definición más amigable del concepto que puede ser entendida no sólo en el ambiente científico sino también por el público en general.

En 1993 el Consejo Británico para el Bienestar de los Animales de Granja publicó las cinco libertades de los animales de Granja (FAWC 1993). Estas describen las condiciones mínimas en que los animales debieran ser mantenidos y reemplazan a las recomendaciones estipuladas previamente por el Consejo de Brambell (1965), las cuales sólo hacían referencia a que el animal tuviese espacio suficiente para moverse libremente, darse vuelta, acicalarse, levantarse y echarse y extender sus extremidades. Las cinco libertades han sido utilizadas como un acercamiento básico al momento de evaluar bienestar animal. Estas consideran:

- Libre de sed, hambre y malnutrición,

- Libre de incomodidad,

- Libre de dolor, injurias y enfermedad,

- Libertad de expresar su comportamiento normal y

- Libre de miedo y distrés

Estas libertades identifican los elementos necesarios para que el animal pueda promover su estado de bienestar y dan un lineamiento básico de las necesidades de los animales. Es así como aparece el concepto de necesidades de los animales, entendiéndose como tal una deficiencia existente en un individuo que puede ser remediada a través de la obtención de un recurso o respondiendo de una manera determinada frente a un medio ambiente específico o un estímulo fisiológico (Fraser y Broom 1990). Si un animal tiene una necesidad, su estado motivacional se ve afectado de manera que genera respuestas conductuales y fisiológicas que le permiten satisfacer esa necesidad o requerimiento específico (Broom 1991).

Muchas veces, debido a condiciones inadecuadas de manejo ambiental y social, los equinos no son capaces de satisfacer sus necesidades, quedando en un estado motivacional muy alto, lo que puede traer consecuencias como la 
aparición de conductas no deseadas. Esto causa, además, un estado de ansiedad y frustración crónica, con lo cual se afecta su estado de bienestar. Wiepkema y Koolhaas (1993) indicaron que los estereotipos son conductas indicativas de un problema de bienestar pasado o presente en el animal. Algunos autores (Broom y Johnson 1993, Laidlow 2001) ${ }^{1}$ sugieren que cualquier nivel de estereotipia representa un problema de bienestar, mientras que otros describen una relación inversa donde a mayor nivel de estereotipia menor estado de bienestar (Dawkins 1990) y, por último, otros han propuesto ciertos porcentajes máximos aceptables como 5\% (Wiepkema y col 1983) o 10\% (Broom 1991) de individuos con estereotipias dentro de una población. Estas conductas han sido utilizadas para evaluar bienestar animal de diversas formas.

La preocupación por las conductas estereotipadas tiene relación con el efecto deletéreo que estas pueden tener sobre el animal y su rendimiento, ya sea en áreas como la reproducción, salud o deporte. Se ha podido establecer que al menos un $68 \%$ de las situaciones que favorecen la presentación de estereotipias son también causales de un deficiente bienestar (Mason y Latham 2004), y pueden ser consideradas como indicador de éste (Parker y col 2008). Para poder utilizar correctamente la estereotipia como indicador de pobre bienestar hay que tener claridad en el mecanismo de su desarrollo. Mason y Latham (2004) sugieren que en aquellos casos en que la conducta ha pasado a ser un hábito controlado a nivel central o cuando posee características autísticas en el control de ésta, posiblemente no será un buen indicador de bienestar, ya que pueden persistir a pesar de realizarse cambios adecuados en el ambiente del animal.

Además del sufrimiento mental que pueden presentar los animales con conductas anormales, se han mencionado otras consecuencias físicas y fisiológicas que acompañan a estas conductas y que también tienen implicancias sobre el estado de bienestar de los equinos. Entre ellas se pueden mencionar la pérdida de condición corporal, problemas de salud asociados (cólico, úlceras gástricas, problemas podales y ortopédicos entre otros), dolor crónico, automutilaciones, elevación de los niveles de cortisol, etc. Todos estos problemas impiden de una forma u otra que el equino se pueda adaptar de forma adecuada a su medio ambiente y pueden ser evaluadas y medidas de manera objetiva y científica.

Por otra parte, es importante recordar que muchas de las medidas que se han tomado en el pasado y que se siguen utilizando para el control de las conductas no deseadas en los equinos también tienen un efecto deletéreo sobre el estado de bienestar del animal, como es el caso del uso de collares antiaerofagia, cirugías, restricción del movimiento y del espacio, etc.

Laidlow R. 2001. The state of the ark: A review of conditions at the Granby Zoo. Available at: www.wspa.ca/reports/granbyzoo.html

\section{CONDUCTAS ESTEREOTIPADAS}

Mason (1991) describe las conductas estereotipadas como patrones de comportamiento invariantes y repetitivos, que no tienen una meta o función obvia. Los estereotipos pueden generarse en aquellos animales enfrentados en su medio ambiente a problemas sin solución (como el encierro en una pesebrera) (Waters y col 2002). Hay que tener en cuenta otras causales de este tipo de conductas, tales como daño cerebral, condiciones psiquiátricas, o efecto de alguna droga como la d-anfetamina (Robbins 1976). En el caso de las personas, las conductas estereotipadas han sido relacionadas con psicopatologías, siendo éstas un componente característico de la esquizofrenia y el autismo temprano (Dantzer 1986). Es por esto que, previo a diagnosticar una conducta como estereotipada, se hace esencial la exclusión de cualquier factor de tipo patológico, que pudiese originar un cambio conductual en el animal (Mills y col 2005). En éstos, las primeras descripciones de estereotipias se realizaron en equinos y en animales silvestres en cautiverio en la década de 1960 y luego en animales de granja mantenidos en sistemas altamente intensivos de producción (Dantzer 1986). En años recientes se ha estimado que las conductas estereotipadas son realizadas por cerca de 85 millones de animales, incluyendo de granja, laboratorio y zoológico (Mason y Latham 2004), siendo los ungulados los más afectados (Bergeron y col 2006).

En el caso de los equinos, a este tipo de conductas se les denomina comúnmente "vicios de pesebrera". Este término es incorrecto, ya que le otorga al equino la capacidad de tomar una decisión de tipo moral, actuando o no de una manera inadecuada (Houpt 1986, Houpt y McDonell 1993). Como la mayoría de estos "vicios" son consecuencia del manejo o estabulación entregados por el hombre, resulta ilógico utilizar dicho término (Broom y Fraser $2007^{\mathrm{a}}$ ). Se asume que los estereotipos no son producto de una selección natural, ya que no representan un beneficio para el individuo y, por otra parte, involucran un costo en términos de energía para éste (Mason 1991). Se considera más bien que ellos son el resultado de un cambio neurológico en respuesta a un estrés crónico (McBride y Hemmings 2004, 2005, 2009).

La manera en que se presente una conducta estereotipada depende de la especie y, dentro de cada especie, pueden existir variaciones entre individuos, especialmente en lo que se refiere a repetibilidad y flexibilidad del patrón conductual (Mason 1991). Por otra parte, el contexto en que se desarrollan las estereotipias está generalmente ligado de manera física y temporal a ambientes subóptimos, los que generan situaciones de frustración, miedo o estrés, restricción física y falta de estimulación (Mason 1991). Dentro de los factores físico-ambientales se encuentran la restricción de la alimentación y de la disponibilidad de uso del tiempo de los individuos (Kiley-Worthington 1987). En el caso de los cerdos, por ejemplo, se sugiere que la 
aparición de conductas estereotipadas sería una respuesta a la restricción de movimiento y subsecuente pérdida de control sobre su ambiente (Cronin 1985). Los equinos a pastoreo utilizan alrededor de 18 horas diarias en actividad de forrajeo, lo cual se ve drásticamente disminuido de 2 a 4 horas en sistemas de estabulación (Boyd y col 1988). Por otra parte, al ser mantenidos en confinamiento en pesebreras existe restricción de espacio y un ambiente bajo en estímulos (Kiley-Worthington 1987). En el contexto social, algunos puntos críticos son el destete temprano, la falta de contacto social y las altas densidades, con un excesivo contacto entre individuos (Kiley-Worthington 1987, Canali y Borroni 1994). A pesar de que los factores ambientales parecen jugar un rol importante en la presentación de estereotipias, no todos los equinos mantenidos dentro de un mismo sistema llegan a presentarlas (Mills y Riezebos 2005).

Entre las posibles causales de estereotipias se ha sugerido el factor de heredabilidad (Vecchiotti y Galanti 1986). A pesar de no existir aún evidencia de esto, se podría heredar la tendencia o predisposición a realizar una estereotipia y no la conducta en sí, la cual sólo se hace evidente bajo ciertos factores de riesgo presentes en el ambiente del animal (Kiley-Worthington 1987, Mills y col 2005). Por otra parte, se ha propuesto una relación entre temperamento y estereotipias, siendo aquellos equinos de temperamento reactivo mayormente predispuestos a realizar estas conductas (Kiley-Worthington 1987). Se han mencionado también como posibles factores causales de estereotipias el aprendizaje social y observacional, aunque no existe evidencia que avale dicha teoría (Murphy y Arkins 2007). En el caso que las conductas estereotipadas funcionaran, a lo menos en parte, como un mecanismo de satisfacer ciertas necesidades que se ven frustradas dentro de un ambiente subóptimo o como estrategia para reducir los niveles de ansiedad, entonces podría ser beneficioso para el animal aprender a realizar estas conductas a través de la observación de individuos más experimentados (Nicol 1999). Esto más bien podría tratarse de un fenómeno de facilitación social, donde se hace evidente una conducta ya establecida en el animal ante la presencia de otro animal que se encuentre realizándola (Mills y col 2005). La mayoría de los estudios se han enfocado en la asociación de algunos factores de manejo y la presentación de conductas estereotipadas, dejando de forma secundaria la relación con factores biológicos. Mills y col (2002), en cambio, estudiaron la relación de estas conductas con sexo, edad y color de capa, encontrando que la prevalencia variaba de forma significativa de acuerdo a sexo (mayor en hembras y machos castrados), a edad (mayor en equinos de 2 años) y no de acuerdo al color de la capa. Por otra parte, McBride y Hemmings (2009) sugieren que las estereotipias son el resultado de cambios neurológicos como respuesta a un estrés crónico.

Gran parte de los conocimientos respecto a esta área provienen de estudios realizados con fármacos, los cuales muestran que drogas psicoestimulantes son capaces de generar conductas estereotipadas en animales de laboratorio, aves y animales de granja (Deviche 1985, Dantzer 1986). La característica común entre estas drogas (anfetaminas, apomorfina) es que interfieren en el metabolismo de las catecolaminas (dopamina y norepinefrina) a nivel cerebral (Dantzer 1986). Se ha relacionado las estereotipias, farmacológicamente inducidas a vías dopaminérgicas en el área nigrostriatal (una de las cuatro vías dopaminérgicas de gran importancia) (Mason 1984). Esta zona es responsable de la iniciación del movimiento, al ser parte del sistema del loop motor del ganglio basal. Aunque las vías dopaminérgicas estuvieran involucradas en las conductas estereotipadas, es importante tomar en cuenta que estos neurotransmisores no actúan de manera independiente, existiendo otros neurotransmisores involucrados en su modulación como serotonina, norepinefrina y péptidos opioides (Dantzer 1986).

En equinos con conductas estereotipadas, específicamente aerofagia, McBride y Hemmings (2005) demostraron la presencia de altas densidades de receptores a dopamina D1 y D2 a nivel del núcleo accumbens, en relación a equinos que no presentaban la conducta. Este incremento en la densidad de estos receptores en el área terminal de la vía messoaccumbens, más una regulación aumentada de los autorreceptores D2 en la porción donde se proyecta esta vía, fue interpretada por Cabib y col (1998) como una facilitación de la vía de transmisión dopaminérgica en el mesoaccumbens. El sistema dopaminérgico nigrostriatal parece ser una estructura clave en la formación de hábitos conductuales, por lo que aquellos animales que presentan conductas estereotipadas pudieran tener una fisiología de la dopamina alterada en esta área del cerebro como consecuencia de la prolongada actividad de ésta (McBride y Hemmings 2005). En otros estudios que avalan la teoría de una relación entre dopamina y estereotipias se ha probado que la administración de agonistas de dopamina inducen estas conductas (Cooper y Dourish 1990), mientras que la administración de antagonistas disminuye significativamente la presentación de ellas (Kostal y Savory 1994).

La vía dopaminérgica, a nivel del mesoaccumbens, es considerada el centro neuronal primario para la iniciación y control de la fase apetitiva de las conductas con objetivos específicos, ya sea a través de la obtención de lo que el animal percibe como positivo o recompensatorio o evitando lo que percibe como aversivo (McBride y Hemmings 2004). Esto significa que una sobrerregulación de este sistema pone al animal en un estado motivacional aumentado con respecto a cualquier conducta orientada a alcanzar un objetivo deseado (Ikemoto y Panksepp 1999). Al utilizar el modelo motivacional sicohidráulico de Lorenz (1950) o el de Hughes y Duncan (1988) (figura 1), nos estaríamos enfrentando a equinos que quedan estancados en la fase apetitiva de la conducta sin poder lograr la fase consumatoria de ésta. A modo de ejemplificar la fase apetitiva y consumatoria de una conducta, Manning y Stamp-Dawkins (1995) explican que un equino pastando 


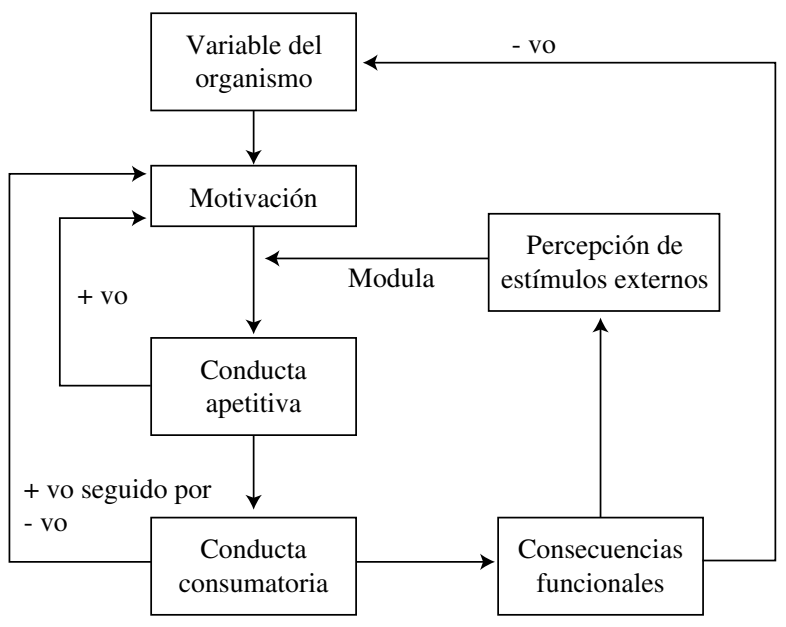

Figura 1. Modelo propuesto por Hughes y Duncan (1988), muestra la separación de la conducta en una fase apetitiva y una consumatoria con sus respectivos sistemas de retroalimentación positivo y negativo.

Model proposed by Hughes and Duncan (1988), showing the division of behaviour into appetitive and consummatory phases with respective positive and negative feedback mechanisms. en el campo literalmente tiene el alimento a sus pies, en cuyo caso la fase apetitiva o de búsqueda es muy corta o no existente y la fase consumatoria de la conducta, o de logro del objetivo, en éste caso la ingesta sería casi continua, de no ser por un descanso de alrededor de una hora cuando el animal está satisfecho.

$\mathrm{Si}$ el ambiente en que se mantiene el animal no le permite lograr el objetivo de su conducta (fase consumatoria), éste se mantiene en un estado de alta motivación dirigido hacia la fase apetitiva de la conducta, resultando en un número limitado de conductas apetitivas realizadas de manera repetida, pudiendo ser ésta la base de la conducta estereotipada (figura 2) (McBride y Hemmings 2004).

Dentro de los métodos diagnósticos de desórdenes conductuales, es importante recolectar la mayor cantidad de información en relación a la historia, rendimiento, manejo y entrenamiento del animal. Es recomendable realizar un chequeo físico teniendo en mente el problema conductual y una observación directa del animal en su medio. La toma de muestras a través de videos continuos o por intervalos es de gran ayuda, ya que las conductas se pueden revisar en detalle, asociar a manejo o situaciones

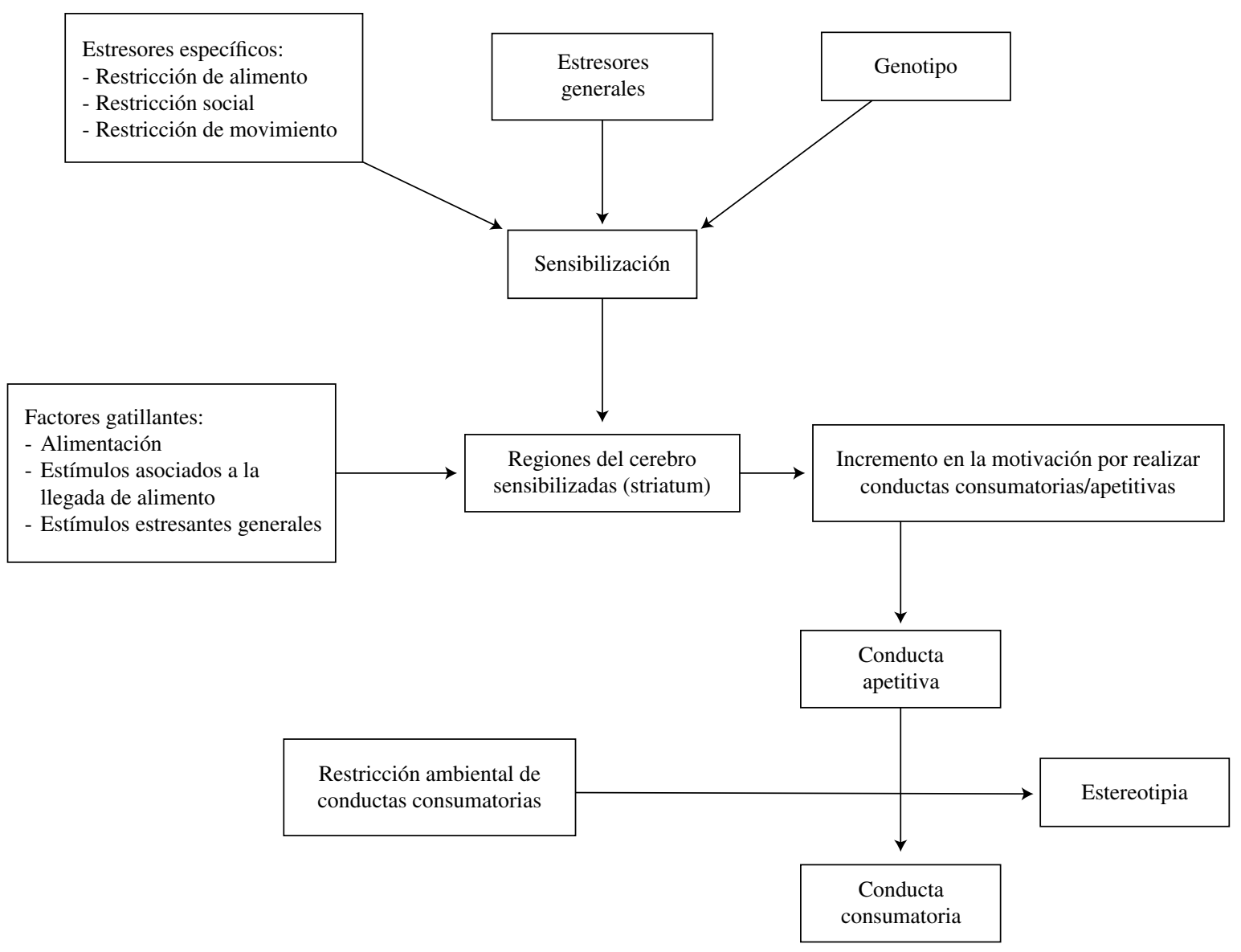

Figura 2. Modelo propuesto por McBride y Hemmings (2004) de las posibles causales de estereotipias. Model proposed by McBride and Hemmings (2004) on the possible causal factor of stereotypic behaviour. 
puntuales, registrar hora del día en que ocurren, su duración y frecuencia (McDonnell 2005).

Aquellas conductas reconocidas como estereotipadas propiamente tal en el equino, comprenden aerofagia con o sin fijación de los incisivos (crib-biting, windsucking), caminar en la pesebrera (pacing, stall-walking) y balanceo del tren anterior (mal del oso o weaving) (McBride y Hemmings 2005, Ninomiya y col 2007). La prevalencia de las tres conductas mencionadas varía entre un 0,3 y $12,3 \%$, dependiendo de la raza, actividad y condiciones de manejo a las que son sometidos (McGreevy y col 1995a, McBride y Long 2001, Christie y col 2006). Otros autores como Mills y col (2005) también incluyen dentro de las estereotipias conductas como sacudir la cabeza (headshaking) y manoteo (pawing). A continuación se analiza cada una de ellas en relación a factores causales, consecuencias sobre el equino y posible tratamiento.

\section{AEROFAGIA CON O SIN FIJACIÓN}

Durante esta conducta el equino se fija a un objeto con los incisivos superiores (generalmente cerco o puerta de la pesebrera), retrae el cuerpo con fuerza y contrae los músculos del cuello, ingresando aire a la porción craneal del esófago, emitiendo un ruido característico (Houpt 1986, Fraser 1992, Redbo y col 1998, Nicol 2000) (figura 3). A medida que la conducta va evolucionando se puede hacer más compleja, tal como lamer el objeto antes y después de la fijación (Nicol 2000). Por otra parte, algunos tragan aire sin necesidad de fijación, especialmente cuando se eliminan las superficies aptas para esto (Houpt 1986, Fraser 1992). A estos equinos se les llama "tragadores de aire" (Fraser 1992), término que puede confundirse con problemas de neumovagina en yeguas (Houpt 1986). Esta actividad puede observarse en equinos que se encuentran solos en la pesebrera o en algunos casos en sincronía con otros caballos (Fraser 1992), pudiendo dedicarle el animal en promedio un $27 \%(1,9-74,6 \%)$ del tiempo a esta actividad (Ninomiya y col 2007).

La aerofagia se clasifica dentro de las estereotipias de tipo oral o ingestiva, con un patrón diurno definido, presentándose de manera postprandial, con una causal ambiental (McBride y Hemmings 2005). McGreevy y col $\left(1995^{\mathrm{a}}\right)$ reportaron prevalencias de un 9,4\%, 9,5\% y $3,9 \%$ para equinos utilizados en adiestramiento, prueba completa y enduro respectivamente. En los Fina Sangre de Carrera, McBride y Long (2001) reportan una prevalencia de 3,8\%, mientras que para el Caballo Chileno Muñoz y col (2009) reportaron una prevalencia de un $2 \%$ en Caballos Chilenos estabulados.

Es importante diferenciar la aerofagia de otra condición clínica, tal como el defecto congénito del cuarto arco bronquial, en el que los animales muchas veces son descritos como tragadores de aire por los propietarios. El diagnóstico clínico de este defecto se realiza por palpación externa y endoscopía, en donde se puede comprobar hipoplasia o ausencia de los músculos cricotiroideo y cricofaríngeo, los cuales se encuentran presentes y normales en el equino con aerofagia estereotipada (Marsden 2002, 2007).

La aerofagia se ha relacionado también con una serie de problemas gastrointestinales (Nicol y col 2002), pudiendo tratarse más bien de una manifestación o consecuencia de éstos más que de una causal adicional, ya que podría ser una conducta que genera una sensación placentera a un animal que sufre de malestar gastrointestinal (Houpt 1986). Esta sensación de placer estaría dada, por ejemplo, por la aerofagia como un potencial medio de producción de saliva para contrarrestar el efecto de acidificación a nivel estomacal e intestino grueso, producto de dietas muy ricas en almidón (Nicol y col 2002, Hemmings y col 2007). Es importante recordar que los equinos sólo producen saliva como respuesta a la masticación, y no como respuesta anticipada a la alimentación. La cantidad de saliva producida va a depender del contenido de agua,

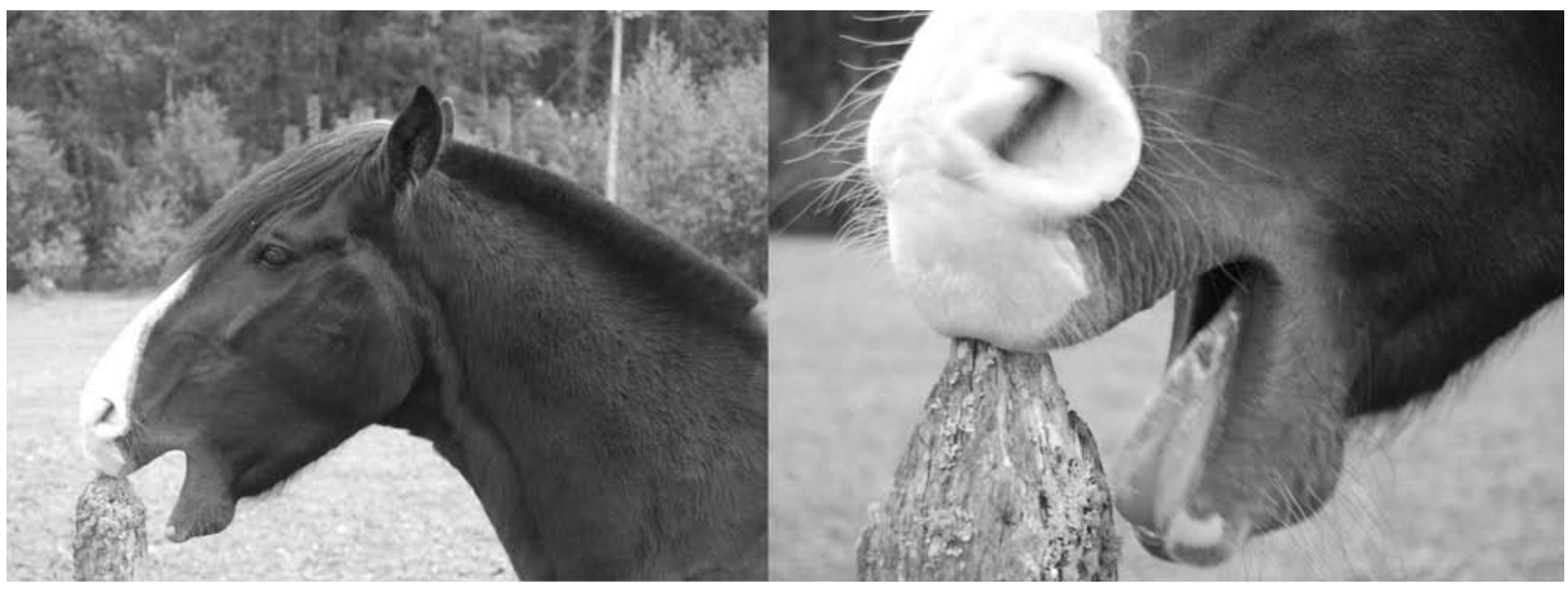

Figura 3. Fotografía de equino realizando la conducta estereotipada de aerofagia sobre un cerco. Photography of a horse performing crib-biting behaviour on a fence. 
tiempo de masticación y naturaleza del alimento (Harris y Arkell 2005). Raciones con un alto porcentaje de concentrado disminuyen la cantidad de saliva producida y con esto la lubricación del bolo de alimento y la alcalinización a nivel de estómago e intestino grueso, favoreciendo la presentación de úlceras gástricas.

Por otra parte, estudios neurobiológicos indican que en aquellos equinos que presentan aerofagia existiría una densidad mayor de receptores a dopamina que en aquellos que no la realizan. Se cree que los caballos que realizan la estereotipia tendrían modificaciones a nivel de las vías dopaminérgicas y que el desarrollo de esta conducta estaría relacionado con cambios dentro del sistema motivacional del animal (McBride y Hemmings 2005). En situaciones de estrés, anfetaminas y apomorfina son estimuladores de liberación de dopamina a nivel del sistema nervioso central, pudiendo causar patrones estereotipados de conducta. Estas conductas se pueden revertir utilizando bloqueadores de dopamina, como por ejemplo haloperidol, pero al parecer el mecanismo no sería tan simple, ya que antagonistas de opioides también pueden tener el mismo efecto. Parece ser que el sistema dopaminérgico se encuentra vinculado con sistemas de transmisión de encefalinas y endorfinas (Dodman y col 1987). Algunos autores, como Cabib y col (1984), postulan que la alta liberación de opioides endógenos, como respuesta a estresores recurrentes, puede llevar a una alteración de la sensibilidad de los receptores dopaminérgicos, cambio que estaría involucrado en los posteriores cambios conductuales.

La dopamina juega un rol importante como reforzador del aprendizaje de nuevas conductas (Phillips y col 2003), por lo cual estos cambios en el sistema motivacional luego del desarrollo de la aerofagia podría afectar el aprendizaje de estos individuos (Ninomiya 2007). Por otra parte, se ha reportado que equinos con aerofagia requirieron de un mayor número de pruebas y respuestas para poder extinguir o mantener respectivamente una respuesta operante, que aquellos equinos que no presentaban la conducta (Hemmings y col 2007, Parker y col 2008). Se sabe que disfunciones a nivel del ganglio basal se expresan a través de problemas de aprendizaje, por lo que si la aerofagia fuera sólo una consecuencia de un malestar gástrico, los equinos que presentan esta conducta no debieran presentar signos de disfunción de dicho ganglio (Hemmings y col 2007). Probablemente se requiera de un mayor número de estudios para poder entender la relación entre estereotipias y habilidad de aprendizaje (Ninomiya 2007).

Dentro de los factores de riesgo asociados a la presentación de aerofagia se encuentran el contacto con otros caballos, el tipo de cama (de paja), el número de horas a pastoreo y el tipo de caballo (Christie y col 2006). Algunos consideran esta conducta como "contagiosa", a pesar que esto no se ha comprobado (Houpt 1986). Podría existir también un factor genético involucrado, ya que algunas familias de caballos Fina Sangre de Carrera parecen tener predisposición a esta conducta, mientras que rara vez es vista en razas de tiro y ponis (Fraser 1992). Sin embargo, es importante tener en cuenta que los sistemas de manejo de estas razas son muy distintos.

Una de las consecuencias innegables de la aerofagia con fijación es el excesivo desgaste de los incisivos superiores (Houpt 1986), pudiendo incluso perderse el contacto entre éstos y los incisivos inferiores al momento de la oclusión, haciéndose muy dificultoso el pastoreo (Fraser 1992). Lo anterior puede llevar a una disminución significativa de la condición corporal y riesgo de ingestión de astillas. Los músculos del cuello también pueden sufrir una hipertrofia (Fraser 1992), con los consecuentes dolores musculares en esta zona. Otra consecuencia de la aerofagia es la dilatación del estómago en aquellos casos en que el aire pasa más allá del esófago, con los subsecuentes episodios de cólico (Fraser 1992).

Existen variados métodos para controlar la aerofagia, desde simples métodos mecánicos hasta complejos procedimientos quirúrgicos y terapias conductuales. El método más simple es la aplicación de un collar, el cual se ajusta alrededor del cuello por detrás de la nuca. $\mathrm{Al}$ arquear el caballo el cuello para tragar aire, el collar ejerce presión produciéndole dolor, dificultando así la realización de la estereotipia. Cuando un collar liso no funciona, se ha descrito el uso de collares con puntas (Houpt 1986), los cuales deben ser removidos a la hora de la alimentación. Existen algunos collares que presentan un receso a nivel de la tráquea, los que pueden ser utilizados sin riesgo (Fraser 1992). También se emplean frenos gruesos de madera o goma para impedir que el equino cierre la boca, los cuales pueden ser efectivos en algunas ocasiones (Fraser 1992). En general estos métodos son considerados poco adecuados desde el punto de vista del bienestar animal, además de sólo restringir la conducta sin eliminar la causal de ésta. Un estudio realizado por McBride y Cuddeford (2001) demostró que el uso del collar antiaerofagia provocaba estrés, encontrando niveles de cortisol significativamente elevados en los equinos en que se utilizaban, mientras que McGreevy y Nicol (1998) reportaron que caballos a los cuales se les impedía la conducta mediante el uso de estos collares durante 24 horas, reanudaban la conducta con mayor intensidad una vez removido el collar.

Otro método para controlar la aerofagia es el diseño de pesebreras sin superficies horizontales donde el caballo pueda apoyar sus dientes (Fraser 1992). Sin embargo, en este caso algunos equinos utilizan baldes, comederos o a sus propios compañeros como punto de apoyo para realizar la conducta o evolucionan a una aerofagia sin fijación de los incisivos (Boyd 1986, Houpt 1986). Otro método de control es la utilización de golpes de corriente a través de electrificación de los objetos que ocupa el animal para la fijación, actuando esto como un castigo ante el estereotipo (Houpt 1986).

Dentro de los métodos quirúrgicos se han descrito la neurectomía de la rama ventral del nervio espinal accesorio, la miotomía de los músculos ventrales del cuello o una combinación de ambos. Otra técnica consiste en una 
miotomía parcial de los músculos omohyoideo, esternohyoideo y esternotiroideo acompañada de la mencionada neurectomía. El éxito con estas cirugías varía de 0 a $70 \%$ (Houpt 1986).

El médico veterinario debiera considerar si realmente se justifica la utilización de los métodos antes mencionados, ya que ellos atentan contra el bienestar del equino. El hecho de que la conducta sea desagradable para el dueño no es una razón válida para someter al equino a una cirugía o al dolor provocado por el collar o electricidad.

Dentro de los tratamientos conductuales se encuentra la terapia de aversión, basada en teorías de aprendizaje, las que consideran esta conducta como resultado de un aprendizaje maladaptativo (Kiley-Worthington 1987). En este tratamiento el caballo debe aprender a “des-aprender" el hábito adquirido (Fraser 1992). Otra posibilidad de control de la conducta es aumentar el tiempo de forrajeo a través de enriquecimiento ambiental. Winskill y col (1996) desarrollaron un aparato conocido como "equiball", que consiste en un balón que al rodar en el piso de la pesebrera va liberando el alimento a través de agujeros, con lo cual se aumenta el tiempo de consumo del caballo; otra forma de obtener el mismo efecto es a través del uso de redes para el forraje.

El uso de fármacos como terapia también ha sido utilizado. Por una parte, y basado en la teoría de que la causal sería un problema gastrointestinal, se ha utilizado el antibiótico virginiamicina como aditivo en la ración. Se sabe que este antibiótico suprime la producción de ácido láctico en los equinos a nivel de intestino grueso (Rowe y col 1994). En este sentido, Johnson y col (1998) reportaron una dramática reducción de conductas anormales de tipo oral en equinos sometidos a una dieta con un contenido de $225 \mathrm{mg}$ /día de virginiamicina. La relación que podría existir entre el uso de virginiamicina y disminución de la conducta, y que de alguna manera apoyaría la teoría de la causal neurológica, podría deberse a que las raciones altamente palatables producen una liberación de endorfinas a nivel de pituitaria, lo que a su vez genera liberación de dopamina y un aumento de la presentación del patrón conductual anómalo (Dodman y col 1987). La virginiamicina podría actuar disminuyendo la palatabilidad de la ración y con ello la liberación de los mencionados neurotransmisores con la subsiguiente disminución de la conducta.

En base a la teoría de un cambio a nivel de transmisión dopaminérgica y opioidea en el sistema nervioso central, se ha estudiado el uso de fármacos antagonistas al neurotransmisor. Se ha visto que la utilización de naloxona reduce de manera significativa la conducta (McBride y Cuddeford 2001). También se han utilizado otros antagonistas de opioides como naltrexona, nalmefeno y diprenorfina, encontrando resultados similares (Dodman y col 1987).

\section{BALANCEO DEL TREN ANTERIOR}

También conocido como "mal del oso", es una conducta no deseada estereotipada de tipo locomotor, preprandial, de origen social (McBride y Hemmings 2005). Se caracteriza por el balanceo de la cabeza, pudiendo también involucrar cuello y miembros anteriores y en ocasiones miembros posteriores (McGreevy y col 1995', Redbo y col 1998, Mills y col 2005). Ocurre en posición de pie, con la cabeza por sobre la puerta de la pesebrera, a pesar de existir individuos que realizan la conducta en el centro de la pesebrera o a potrero (Cooper y col 2000, Nicol 2000). Frecuentemente se asocia a animales de temperamento nervioso (Fraser 1992), mantenidos bajo sistemas de manejo donde los requerimientos nutricionales y de ejercicio no son satisfechos (Mills y col 2005). Generalmente este balanceo es realizado como una respuesta condicionada frente a una señal, la cual indica la llegada próxima de alimento (McBride y Cuddeford 2001, Ninomiya y col 2007). En un estudio realizado por Cooper y col (2005) se incrementó el número de raciones de alimento concentrado en equinos que presentaban algún tipo de estereotipia, encontrándose que, a pesar de disminuir la presentación de estereotipias orales, el aumento en el número de raciones entregadas se reflejó en un incremento de la presentación del balanceo estereotipado. Los equinos pueden dedicar entre un 0,3 a un $61 \%$ de su tiempo a esta actividad (Ninomiya y col 2007). Se han registrado prevalencias de 7,5\%, $8,3 \%$ y $3,1 \%$ para equinos utilizados en adiestramiento, prueba completa y enduro respectivamente (McGreevy y col $1995^{\mathrm{a}}$ ), y de un 2,6\% para equinos Fina Sangre de Carrera (McBride y Long 2001). En Chile Muñoz y col (2009) reportaron una prevalencia de un $1 \%$ para Caballos Chilenos estabulados.

Dentro de los factores de riesgo asociados al mal del oso, según Christie y col (2006) destacan el uso de cama de paja, número de horas en que el animal trabaja durante la semana, tipo, sexo y el tipo de freno utilizado, aumentando el riesgo con aquellos frenos más agresivos. El mismo estudio destaca que el uso de frenos más agresivos se utiliza por lo general en animales de temperamento más nervioso, lo cual podría representar un incremento en su restricción física, reduciendo el control del animal sobre su ambiente, lo cual le causaría distrés. Ninomiya y col (2007), por su parte, proponen una relación entre frustración alimenticia como resultado de una dieta pobre en fibra y la presentación de esta conducta.

Nicol (2000) sugiere que esta conducta puede surgir como consecuencia de un escape frustrado, ya que se ha observado que algunos caballos comienzan a realizar la estereotipia cuando sus vecinos son removidos de la pesebrera, a lo cual responde con un esfuerzo por recobrar contacto social. Mills y col (2005) refuerzan dicha teoría sosteniendo que esta conducta es menos común en establecimientos con más de 75 equinos, donde se les permite el contacto visual y táctil con sus coespecíficos. Algunos propietarios indican como causa de esta esterotipia la imitación, y evitan mantener en el mismo establo a equinos que presentan la conducta con aquellos que no la presentan (McBride y Long 2001). 
Una consecuencia del mal del oso es el desgaste energético consecuente al movimiento involucrado en la estereotipia, lo cual lleva a pérdida de peso y que los animales estén físicamente exhaustos (Fraser 1992, Mills y Riezebos 2005). En aquellos equinos que además balancean los miembros anteriores puede ocurrir un desgaste disparejo de los cascos (Ninomiya 2007). También se menciona desarrollo asimétrico de masas musculares a nivel de cuello, utilización ineficiente del alimento y exacerbación de problemas ortopédicos concomitantes (Mills y Riezebos 2005).

Una vez adquirida la conducta es muy difícil eliminarla. Dentro de los sistemas utilizados para ello están la estabulación, con el equino atado con riendas cruzadas para limitar el movimiento lateral (Fraser 1992) o cerrar la parte anterior de la pesebrera con barras para evitar el balanceo de la cabeza por sobre la puerta. Este último método no ha tenido buenos resultados, ya que algunos equinos realizan la conducta en el centro de la pesebrera o comienzan movimientos de balanceo del tren posterior. McBride (1996) no pudo reducir la ocurrencia de esta conducta utilizando las barras en 3 caballos con mal del oso que incluyó en su estudio.

$\mathrm{El}$ enriquecimiento ambiental ha sido utilizado para disminuir esta conducta, ya sea modificando la pesebrera a través de la incorporación de espejos o ventanas, permitiendo la interacción social (Cooper y col 2000) o mediante imágenes (Mills y Riezebos 2005). Estos últimos autores utilizaron 3 tratamientos, los que consistían en 3 póster $(0,9 \mathrm{~m}$ x $0,6 \mathrm{~m}$ ), un póster con la figura tamaño real de la cabeza de un caballo, otro con la misma imagen, pero pixelada y reorganizada y uno en blanco. La imagen de la cabeza de un caballo tamaño real disminuyó significativamente la presentación del mal del oso. Según los mismos autores, algunas conductas podrían ser moduladas a través de la entrega de estímulos visuales bidimensionales, sin la necesidad de un procesamiento más complejo en tres dimensiones. En todo caso, lo ideal es que los equinos que realizan esta conducta sean mantenidos a pastoreo (Fraser 1992).

\section{DEAMBULAR ESTEREOTIPADO}

También conocido como "caminar en la pesebrera", se caracteriza por un deambular en círculos de manera constante, en una misma dirección dentro de la pesebrera, alternando direcciones o en forma de ocho (Redbo y col 1998). Al aumentar el espacio dentro de la pesebrera, ellos incrementan también el espacio para realizar la conducta, a veces efectuando circuitos más complejos (Fraser 1992). Esta conducta se clasifica como estereotipada locomotora de presentación preprandial. En el Reino Unido se reportaron prevalencias de 3,8\% para equinos de adiestramiento, 3,6\% en equinos de prueba completa y $5,5 \%$ en enduro (McGreevy y col $1995^{\mathrm{a}}$ ), mientras que para equinos Fina Sangre de Carrera McBride y Long (2001) reportaron una prevalencia de $1,2 \%$, mientras que para el Caballo Chileno se ha reportado una prevalencia del 8\% (Muñoz y col 2009).
En los équidos mantenidos en parques zoológicos también se ha descrito esta estereotipia. Algunas de las posibles causas serían obstáculos a la actividad motora, especialmente en animales altamente activos mantenidos en un recinto limitado, falta de estímulos en el medio ambiente que puedan distraer su atención hacia otra actividad, anticipación a la llegada de alimento. También como respuesta de escape ante la presencia de personas o ante individuos dominantes dentro del recinto y estrés por separación o cuando puede oler, ver o escuchar a antiguos compañeros (Boyd 1986).

Es importante realizar un diagnóstico diferencial entre el deambular estereotipado y el caminar en círculo, mostrado este último como signo clínico en animales que padecen de enfermedad cerebral mórbida. En el deambular estereotipado, el caballo puede cambiar de dirección, además permanece atento al medio y al manejo rutinario al que es sometido. Por otra parte, no presenta asimetría en la sensibilidad y expresión facial (Marsden 2002, 2007).

Es común apreciar el deambular estereotipado en sementales mantenidos con fines reproductivos en potrerillos pequeños. Un ejemplo de esto lo revela un estudio en que un semental Przewalski mantenido solo ocupaba un 4\% de su tiempo en esta actividad (Boyd 1986).

Los efectos de esta conducta sobre el caballo van desde pérdida de condición física hasta dolores crónicos de columna, sobre todo en aquellos mantenidos en pesebreras pequeñas, los cuales requieren de círculos más cerrados para realizar la conducta (Fraser 1992). También se puede observar en algunos un desgaste disparejo de los cascos (Ninomiya 2007).

Dentro de los tratamientos utilizados para esta conducta está la estabulación con el caballo atado, lo que evita la realización de la estereotipia pero no acaba con el problema básico (Fraser 1992). Muchas veces el uso de este método restrictivo lleva a que los caballos pasen de un deambular estereotipado a un balanceo del tren anterior. El uso de enriquecimiento ambiental en la pesebrera es una alternativa que puede disminuir la conducta (Houpt 1986).

\section{OTRAS CONDUCTAS NO DESEADAS EN EQUINOS}

\section{CONDUCTAS REDIRIGIDAS}

Son aquellas conductas anormales dirigidas hacia un objeto diferente de la fuente del estímulo (McGreevy 2004). Es un tipo de conducta de conflicto, donde se activan dos sistemas motivacionales, pero sólo uno de ellos logra expresarse dirigiéndose a un objeto inapropiado (Barnard 2004 ${ }^{\mathrm{a}}$ ).

Lignofagia. Este patrón de comportamiento, donde el equino mastica e ingiere madera, es bastante flexible y muchas veces precede a la conducta de aerofagia (Nicol 1999). Durante la lignofagia el equino se fija a objetos horizontales 
(como la puerta de la pesebrera), para arrancar pedazos de madera, los cuales muchas veces son ingeridos (Redbo y col 1998). La principal causa de esta conducta sería la falta de fibra en la dieta, encontrándose un aumento en la presentación de ella en equinos con dietas altas en concentrados u otros alimentos peleteados (Boyd 1986, Krzak y col 1991). Esto transformaría a esta conducta en algo funcional para el caballo, permitiéndole la obtención adicional de fibra (Redbo y col 1998). Al parecer, aunque los requerimientos energéticos del equino sean satisfechos con la dieta entregada, éstos sienten la necesidad de realizar la conducta de forrajeo, aunque ello signifique trabajar para conseguirlo (Houpt 2005).

Coprofagia. A pesar que la coprofagia es una conducta normal en equinos hasta el primer mes de edad, generalmente no ocurre en adultos (Waring 2003). Es común que potrillos consuman materia fecal de sus madres, por lo que se ha sugerido la existencia de una discriminación del material fecal materno en base a feromonas (Crowell-Davies y Caudle 1989). Se ha sugerido que esta conducta le permite al potrillo adquirir microorganismos intestinales, los que le serán de utilidad durante el cambio de una dieta basada en leche a una basada en forraje (Waring 2003). También se ha demostrado que la materia fecal contiene altos niveles de ácido desoxicólico, del cual son deficientes los potrillos, existiendo evidencia de que éste provee protección contra enteritis en la fase juvenil de algunos vertebrados (Crowell-Davies y Caudle 1989). En el equino adulto esta conducta parece desencadenarse por una falta de estímulos orales, tal como cuando los animales son mantenidos en áreas sin forraje o con dietas altas en concentrados (Boyd 1986).

Agresión. Existen variados tipos de esta conducta, tal como la agresión afectiva relacionada con una respuesta autonómica que incluye interacciones simpático-adrenales. En ella el animal muestra signos a través del lenguaje corporal, el que siempre incluye una amenaza, pudiendo llegar al ataque propiamente tal cuando no se toman las medidas apropiadas. Dentro de la agresión afectiva se encuentran aquellas inducidas por dolor, por miedo, interacciones entre machos, por dominancia, protectiva, maternal, aprendida y la redirigida, que es la que en este caso interesa (Beaver 1986, Fraser 1992).

Por otra parte, se encuentra la agresión no afectiva, la cual no está usualmente asociada a signos de amenaza, siendo las situaciones en que ocurre generalmente predecibles. Dentro de este grupo se encuentran las agresiones durante el juego y las asociadas a la reproducción (Beaver 1986). Existen también otras causas de agresión, tal como la observada en animales irritables, hipertestosteronismo en hembras, factores genéticos, disfunción cerebral y automutilación.

Cuando la agresión es redirigida, el equino se encuentra estimulado por un aliciente que no puede alcanzar y redirige su hostilidad hacia otro animal, persona u objeto que tenga cerca, considerándose ésta como una conducta anormal reactiva (Fraser 1992). Un ejemplo de este tipo de agresión ocurre cuando un equino hambriento es cepillado, mientras al del lado se le da de comer. Probablemente el caballo, frustrado por no tener alimento, intente morder a la persona que lo está cepillando. Otra circunstancia en la que la agresión puede presentarse es en aquellos mantenidos dentro de un ambiente inadecuado (espacio reducido, mucha luz, ruido, entre otros). Así, cuando una persona trate ingresar a la pesebrera el animal intentará morderlo (Beaver 1986, Houpt 1986). Por lo general esta conducta se da más en machos enteros, quienes exhiben la conducta de amenaza (orejas hacia atrás, incisivos expuestos, labios retraídos y movimiento de cola) seguida rápidamente por la mordida (Fraser 1992).

Esta conducta puede ser controlada a través de condicionamiento negativo, para lo cual el animal debe ser castigado de manera inmediata de realizada la conducta (Fraser 1992).

Automutilación. Puede ocurrir como conducta anormal en equinos altamente estresados (Beaver 1986) y típicamente en aquellos mantenidos aislados (Broom y Fraser 2007b). La automutilación es considerada como una anomalía conductual seria. Los animales afectados pueden morderse los flancos o rozarse contra objetos hasta producirse heridas abiertas. La conducta se caracteriza por la intensidad con que es realizada y por estar en ocasiones acompañada por vocalización (Fraser 1992). Es importante descartar otras causales como neuritis, alergias dérmicas, irritantes como jabón u otros elementos (Beaver 1986).

El otorgarle compañía al individuo o un ambiente más complejo en que se pueda desenvolver puede ser de ayuda en el control de la conducta (Fraser 1992). En algunos casos llega a ser necesario el uso de tranquilizantes para terminar con un episodio de automutilación (Broom y Fraser 2007 ).

Comer la cama. Esta conducta se considera como redirigida, ya que el objetivo primario del equino es el consumo de fibra (forrajeo). Al mantenerse en pesebrera con raciones controladas redirige esta conducta hacia el material de la cama (McGreevy 2004).

\section{CONDUCTAS VACÍAS}

Se refiere a la ocurrencia de una conducta normal sin la presencia del estímulo correcto (Manning y StampDawkins 1995, Barnard 2004 b).

Frotar la cola contra un objeto. Esta conducta no es específica y generalmente puede ocurrir como signología de parasitismo por Oxyuris, infección perianal por hongos, en animales con dolor articular crónico de tarsos, etc. Cuando la conducta persiste sin presencia de una infección es considerada anormal somática (Fraser 1992). 
Los equinos la realizan apoyándose contra un árbol, cerco o construcción, moviendo los cuartos posteriores de manera rítmica. El resultado de esta actividad es pérdida de crin desde la base de la cola (Fraser 1992).

\section{CONDUCTAS DESPLAZADAS}

Este tipo de comportamiento fue descrito por Kortland (1940) y Tinbergen (1951). La principal característica es la aparente irrelevancia ante la situación en la que ocurre (Manning y Stamp-Dawkins 1995) o como una respuesta inapropiada frente a la situación a la que se ve enfrentado el animal (McGreevy 2004). Según Barnard (2004ª), este tipo de conducta conflictiva ocurre cuando se activan 2 centros motivacionales incompatibles entre sí, los cuales no logran expresarse, desplazando esta energía hacia un tercer centro irrelevante ante las circunstancias, resultando en una conducta fuera de contexto.

Acicalamiento anormal. Algunos caballos cuando están ensillados y no se les permite avanzar por un tiempo más prolongado al tolerado, comienzan a arquear el cuello de forma lateral con el fin de acicalarse los flancos o miembros anteriores, respuesta que no es la apropiada frente a la situación (McGreevy 2004).

Manoteo. El manoteo es una conducta normal utilizada para retirar la nieve del pasto o llamar la atención de un potrillo en decúbito. Es considerada como desplazada cuando es realizada de manera vigorosa y repetida como respuesta a un estado de frustración, como puede suceder en caso de tratar de obtener alimento (Fraser 1992, McGreevy 2004), alcanzar a otro animal o cuando se mantienen atados por tiempos largos o tratan de escapar de su pesebrera (Houpt 1986).

Cuando la conducta ocurre sobre superficies duras puede tener como consecuencia lesiones a nivel de casco y tendones (Fraser 1992). Esta conducta ocurre generalmente en equinos que son mantenidos aislados, por lo que podría ser controlada al mantenerlos en grupo a pastoreo (Fraser 1992).

Inmovilidad tónica - Diskinesis tónica. Corresponde a un comportamiento severo de inercia por sumisión, el cual se manifiesta con una inmovilización en una posición o postura determinada (Fraser 1992). Generalmente ocurre como respuesta a una situación de miedo o estrés. Es importante descartar cualquier patología, sobre todo aquellas que causen dolor, las que puedan hacer que el animal se rehúse al movimiento (Fraser 1992).

\section{CONDUCTAS APRENDIDAS}

El aprendizaje en los animales muchas veces no es reconocido y, por lo tanto, es mal interpretado. En algunos casos los equinos adquieren esta conducta de manera accidental al ejecutar una acción que está ligada a un refuerzo positivo, la cual es a veces realizada por el mismo propietario o jinete (McGreevy 2004). Un ejemplo extremo de estas conductas es el caso de cólico psicogénico presentado por un caballo que buscaba compañía humana (Murray y Crowell-Davies 1985).

Los equinos están aprendiendo todo el tiempo, por esto es importante tener en cuenta que "no porque no se tenga la intención de enseñarle algo al caballo quiere decir que no se haga, y no porque no se esté consciente de haberle enseñado algo quiere decir que no se haya hecho" (Hagerbaumer 1995).

Agresión aprendida hacia los humanos. Esto puede ser el resultado de conductas tan simples como la entrega de alimento cuando el equino tiene adoptada una postura agonista. Con el tiempo el equino aprende a asociar la llegada de alimento con la reacción agonista. Esta postura de amenaza puede aumentar cuando la persona castiga al animal y luego se retira, con lo que inconscientemente está recompensando al equino con su partida y reforzando la conducta agresiva (McGreevy 2004).

La agresión también puede ser producto de una respuesta defensiva aprendida. Esto puede ocurrir cuando los equinos son mantenidos en grupo en un área pequeña sin suficiente alimento o agua, teniendo que competir por los recursos disponibles. También puede ocurrir cuando ellos asocian el trabajo con montura a un manejo doloroso y se comporten de manera agresiva frente a la llegada del jinete (McGreevy 2004). Otra causal es la socialización temprana con humanos, lo que puede terminar en un mal-imprinting, acostumbrándose los caballos a morder a las personas como forma de saludo o responder con acercamiento positivo, en vez de alejarse cuando la persona que considera como su cuidador se le acerca (entra en su zona de fuga) (Fraser 1992).

Patear la pesebrera. $\mathrm{Al}$ igual que muchas de las conductas mencionadas, el patear las paredes de la pesebrera podría ser una manera de autoestimularse, ya que lo que el equino busca es escuchar el ruido que producen los cascos contra la pared (Houpt 1986).

Esta actividad puede producir contusiones no deseadas en el pie, además de los evidentes daños a la pesebrera. A veces, el eliminar la cama y utilizar suelos de madera le permite al animal escuchar sus pisadas reduciendo la presentación de la conducta (Houpt 1986).

\section{CONCLUSIONES}

Cabe destacar que existe un gran número de conductas no deseadas en los equinos, pero que sin duda las más estudiadas han sido las estereotipadas, y dentro de ellas la aerofagia con fijación. Esto pudiese deberse a lo llamativo que pueden llegar a ser los repertorios conductuales estereotipados, a su prevalencia y a las consecuencias negativas en términos productivos, de salud y bienestar que ellas tienen sobre el animal. 
$\mathrm{Al}$ enfrentarnos con un caballo que presenta una conducta anormal hay que tener en cuenta que cualquiera sea el origen de ésta (ambiental, social, patológica, etc.), su estado de bienestar se encuentra reducido, ya sea por la causa que genera su presentación, por las consecuencias de su realización o las posibles medidas de control o tratamiento que se adopten.

La identificación de estas conductas por parte del propietario y del médico veterinario, y la correcta asociación de su presentación con factores de riesgos presentes en el sistema permitirán el desarrollo de prácticas de manejo y diseños de recintos adecuados para la mantención de equinos, evitándose la aparición de estas conductas en el futuro.

\section{RESUMEN}

El bienestar animal es un área de investigación de gran relevancia en la actualidad, encontrándose en la literatura una gran variedad de trabajos que evalúan el estado de bienestar de distintas especies a través del uso de indicadores de tipo directo e indirecto. Dentro de los indicadores directos posibles de utilizar cobran cada vez más importancia aquellos de tipo conductual y, dentro de éstos, la presentación de conductas anormales, particularmente por las implicancias físicas, fisiológicas y mentales que este tipo de conducta trae consigo. Existe un gran número de publicaciones que entregan antecedentes sobre las diversas conductas anormales que se pueden observar en equinos, muchas veces haciendo confusa su comprensión y clasificación. La presente revisión tiene por objetivo describir aquellas conductas consideradas anormales o no deseadas en la especie equina, con énfasis en conductas consideradas estereotipadas. Para ello, se aporta información sobre sus causas, formas de presentación, función y posibles métodos de prevención. Por otra parte, se entregan, antecedentes sobre las implicancias que éstas tienen sobre el bienestar de los equinos.

\section{REFERENCIAS}

Barnard C. 2004a . Causation and motivation. In: Barnard C (ed). Animal Behaviour: mechanism, development, function and evolution. Pearson Education Limited, London, UK, Pp 185-195.

Barnard C. 2004 ${ }^{\mathrm{b}}$. Motivation and cognition. In: Barnard C (ed). Animal Behaviour: mechanism, development, function and evolution. Pearson Education Limited, London, UK, Pp 167-176.

Beaver BV. 1986. Aggressive behavior problems. Vet Clin N Am-Equine Pract 2, 635-644.

Bergeron R, A Badnell-Waters, S Lambton. G Mason. 2006. In: Mason G, Rushen J (eds). Stereotypic animal behaviour - fundamentals and applications for welfare. $2^{\text {nd }}$ ed. CABI, Wallingford, UK, Pp 19-49.

Boyd LE. 1986. Behavior problems of equids in zoos. Vet Clin N AmEquine Pract 2, 653-664.

Boyd LE, DA Carbonaro, KA Houpt. 1988. The 24-hour time budget of Przewalski horses. Appl Anim Behav Sci 21, 5-17.

Brambell FWR. 1965. Report of the Technical Committee to Enquire into the Welfare of Animals kept under Intensive Livestock Husbandry Systems. HMSO, London, UK.

Broom DM. 1986. Indicators of poor welfare. Br Vet $J 142,524$.

Broom DM. 1991. Animal welfare: concepts and measurements. J Anim Sci 69, 4167-4175.

Broom DM, K Johnson. 1993. Stress and animal welfare. Chapman and Hall, London, UK.

Broom DM, AF Fraser. 2007 . Abnormal behaviour 1: stereotypies. In: Broom DM, Fraser AF (eds). Domestic animal behaviour and welfare. $4^{\text {th }}$ ed. Oxford University Press, New York, USA, Pp 226-234.

Broom DM, AF Fraser. 2007 ${ }^{\mathrm{b}}$. Abnormal behaviour 2: self directed and environment directed. In: Broom DM, Fraser AF (eds). Domestic animal behaviour and welfare. $4^{\text {th }}$ ed. Oxford University Press, New York, USA, Pp 235-238.

Cabib S, S Puglisi-Allegra, A Oliverio. 1984. Chronic stress enhances apomorphine induced stereotyped behavior in mice: involvement of endogenous opioids. Brain Res 298, 138-140.

Cabib S, L Giardino, L Calza, M Zanni, A Mele. 1998. Stress promotes major changes in dopamine receptor densities within the mesoaccumbens and nigrostriatal systems. Neuroscience 84, 193-200.

Canali E, A Borroni. 1994. Behavioural problems in thoroughbred horses reared in Italy. Appl Anim Behav Sci 40, 74.

Christie JL, CJ Hewson, CB Riley, MA McNiven, IR Dohoo, LA Bate. 2006. Management factors affecting stereotypies and body condition score in noneracing horses in Prince Edward Island. Can Vet J 47, 136-143.

Cooper SJ, CJ Dourish. 1990. An introduction to the concept of stereotypy and a historical perspective o the role of brain dopamine. In: Cooper SJ, Dourish CJ (eds). Neurobiology of stereotyped behaviour. Clarendon Press, Oxford, UK, Pp 1-24.

Cooper JJ, L McDonald, DS Mills. 2000. The effect of increasing visual horizons on stereotypic weaving: implications for the social housing of stabled horses. Appl Anim Behav Sci 69, 67-83.

Cooper JJ, N Mcall, S Johnson, HPB Davidson. 2005. The short-term effects of increasing meal frequency on stereotypic behavior of stabled horses. Appl Anim Behav Sci 90, 351-364.

Cronin GM. 1985. The development and significance of abnormal and stereotyped behaviours in tethered sows. PhD thesis, Agricultural University Wageningen, The Netherlands.

Crowell-Davies SL, AB Caudle. 1989. Coprophagy by foals: recognition of maternal feces. Appl Anim Behav Sci 24, 267-272.

Dantzer R. 1986. Behavioral, physiological and functional aspects of stereotyped behavior: a review and a re-interpretation. J Anim Sci 62, 1776-1786.

Dawkins MS. 1990. From an animals point of view: motivation, fitness, animal welfare. Behav Brain Sci 13, 1-61.

Dawkins MS. 2008. The science of animal suffering. Ethology 114, 937-945.

Deviche P. 1985. Behavioral response to apomorphine and its interaction with opiates in domestic pigeons. Pharmacol Biochem Behav 22, 209-214.

Dodman NH, L Shuster, MH Court, R Dixon. 1987. Investigation into the use of narcotic antagonists in the treatment of a stereotypyc behavior pattern (crib biting) in the horse. Am J Vet Res 48, 311-319.

Duncan IJH, D Fraser. 1997. Understanding animal welfare. In: Appleby MC, Hughes BO (eds). Animal welfare. CABI, Wallingford, UK, Pp 19-31.

Duncan IJH. 1998. Behavior and behavioral needs. Poult Sci 77, 17661772.

FAWC, Farm Animal Welfare Council. 1993. Second report on priorities for research and development in farm animal welfare. Ministry of Agriculture, Fisheries and Food, UK.

Fraser A, DM Broom. 1990. Farm animal behaviour and welfare. Saunders, New York, USA.

Fraser A. 1992. Abnormal and anomalous behaviour. In: Fraser A (ed) The behaviour of the horse. CABI, Newfoundland, Canada.

Fraser D. 2003. Assessing animal welfare at the farm and group level: the interplay of science and values. Anim Welf 12, 433-443.

Hagerbaumer J. 1995. Exploring the equine mind with learning and memory studies. In: The thinking horse. Guelph, Equine Research Centre, Canada.

Harris PA, K Arkell. 2005. How understanding the digestive process can help minimize digestive disturbances sue to diet and feeding practices. In: Harris PA, Mair TS, Slater JD, Green RE (eds). The $1^{\text {st }}$ BEVA and Waltham nutrition symposia, "Equine nutrition for all". Harrogate, UK.

Hemmings A, SD McBride, CE Hale. 2007. Perseverative responding and the aetiology of equine oral stereotypy. Appl Anim Behav Sci 104, 143-150.

Houpt KA. 1986. Stable vices and trailer problems. Vet Clin N AmEquine Pract 2, 623-644. 
Houpt KA, SM McDonell. 1993. Equine stereotypies. Comp Cont Educ Pract Vet 15, 1265-1271

Houpt KA. 2005. Maintenance behaviours. In: Mills D, McConnell S (eds). The domestic horse: the evolution development and management of its behaviour. Cambridge University Press, Cambridge, UK, Pp 94-109.

Hughes BO, IJH Duncan. 1988. The notion of ethological need, models of motivation and animal welfare. Anim Behav 36, 1696-1707.

Ikemoto S, J Panksepp. 1999. The role of nucleus accumbens dopamine in motivated behavior: a unifying interpretation with special reference to reward seeking. Brain Res Rev 31, 6-41.

Johnson KG, J Tyrrell, JB Rowe, DW Pethick. 1998. Behavioural changes in stabled horses given nontherapeutic levels of virginiamycin. Equine Vet J 30, 139-143.

Keiper R. 1986. Social Structure. Vet Clin N Am-Equine Pract 2, 465-486.

Kiley-Worthington M. 1977. Behavioural problems of farm animals. Oriel Press, Stocksfied, England.

Kiley-Worthington M. 1987. Behavioural problems. In: Kiley-Worthington $\mathrm{M}$ (ed). The behaviour of horses in relation to management and training. JA Allen, London, UK.

Kortland A. 1940. Ein übersicht der angeborenen Verhaltungsweisen des mittel europäischen Kormorans (Phalacrocorax carbosinensis), ihre funktion, ontogenetische Entwicklung und phylogenetische Herkunft. Arch Néerl Zool 4, 401-442.

Kostal L, CJ Savory. 1994. Influence of pharmacological manipulation of dopamine and opioid receptor subtypes on stereotyped behavior of restricted fed fowls. Pharm Biochem Behav 48, 241-252.

Krzak WE, HW Gonyou, LMLawrence. 1991. Wood chewing by stabled horses, diurnal pattern and effects of exercise. J Anim Sci 69, 1053-1058.

Lorenz K. 1950. The comparative method in studying innate behaviour patterns. Symp Soc Exp Biol 4, 221-268.

Manning A, M Stamp-Dawkins. 1995. Motivation and decision making. In: Manning A, Stamp-Dawkins M(eds). An introduction to animal behaviour. $4^{\text {th }}$ ed. Cambridge University Press, Cambridge, UK, Pp 71-100.

Marsden MD. 2002. A new perspective on stereotypic behaviour problems in horses. In Practice 24, 558-569.

Marsden MD. 2007. Stereotypic and other behavior problems. In: Reed S, Furr M (eds). Equine Neurology. Blackwell Publishing, London, UK, Pp 373-402.

Mason ST. 1984. Catecholamines and behavior. Cambridge University Press, Cambridge, UK

Mason G. 1991. Stereotypies - a critical review. Anim Behav 41, 1015-1037.

Mason GJ, NR Latham. 2004. Can't stop, won't stop: is stereotypy a reliable animal welfare indicator? Anim Welf 13 Suppl 1, 57-69.

McBride SD. 1996. A comparison of physical and pharmacological treatments for stereotyped behaviour in the horse. In: Duncan IJH, TM Widowski, DB Haley (eds). Proceedings of the $30^{\text {th }}$ international congress for the international society of applied ethology. CSAW, Guelph, Canada.

McBride SD, D Cuddeford. 2001. The putative welfare reducing effect of preventing equine stereotypic behaviour. Anim Welf 10, 173-189.

McBride SD, L Long. 2001. The perception and subsequent management of equine stereotypic behaviour by horse owners: implications for animal welfare. Vet Rec 148, 799-802.

McBride SD, A Hemmings. 2004. Causal factors of equine stereotypy. BSAS occasional publications $n^{\circ} 32, \mathrm{Pp} 35-66$.

McBride SD, A Hemmings. 2005. Altered mesoaccumbens and nigro-striatal dopamine physiology is associated with stereotypy development in a non rodent species. Behav Brain Res 159, 113-118.

McBride SD, A Hemmings. 2009. A neurologic perspective of equine stereotypy. JEVS 29, 10-16.

McDonnell SM. 2005. Is it Psychological, physical, or both? In-Depth: Behavior, AAEP Proceedings 51, Washington, USA.

McGreevy PD, NP French, CJ Nicol. 1995 . The prevalence of abnormal behaviors in dressage, eventing and endurance horses in relation to stabling. Vet Rec 137, 36-37.

McGreevy PD, PJ Cripps, NP French, LE Green, CJ Nicol. 1995 ${ }^{\text {b }}$ Management factors associated with stereotypyc and redirected behaviour in the Thoroughbred horse. Equine Vet J 27, 86-91.
McGreevy PD, CJ Nicol. 1998. The effect of short term prevention on the subsequent rate of crib biting in thoroughbred horses. Equine vet J, Suppl 27, 30-34.

McGreevy PD. 2004. Equine behavior: a guide for veterinarians and equine scientists. Saunders, Elsevier, London,UK.

Mills DS, RD Alston, V Rogers, NT Longford. 2002. Factors associated with the prevalence of stereotypic behavior amongst Throughbred horses passing through auctioneer sales. Appl Anim Behav Sci 78, 115-124.

Mills DS, M Riezebos. 2005. The role of the image of a conspecific in the regulation of stereotypic head movements in the horse. Appl Anim Behav Sci 91, 155-165.

Mills DA, KD Taylor, JJ Cooper. 2005. Weaving, Headshaking, Cribbing and other stereotypies. In Depth: Behavior. AAEP Proceedings, vol 51. Washington, USA.

Muñoz L, J Torres, O Sepúlveda, C Rehhof, R Ortiz. 2009. Frecuencia de comportamientos anormales estereotipados en Caballos Chilenos estabulados. Arch Med Vet 41, 73-76.

Murphy J, S Arkins. 2007. Equine learning behaviour. Behav Process 76, $1-13$.

Murray MJ, S Crowell-Davies. 1985. Psychogenic colic in a horse. J Am Vet Med Assoc 186, 381-383.

Nicol CJ. 1999. Understanding equine stereotypies. Equine Vet J, suppl. 28, 20-25.

Nicol CJ. 2000. Equine stereotypies. In: Houpt KA (ed). Recent advances in companion animal behavior problems. International Veterinary Information Service. Ithaca, New York, USA.

Nicol CJ, HPD Davidson, PA Harris, AJ Waters, AD Wilson. 2002. Study of crib biting and gastric inflammation and ulceration in young horses. Vet Rec 151, 658-662.

Ninomiya S. 2007. Social learning and stereotypy in horses. Behav Process 76, 22-23.

Ninomiya S, S Sato, K Sugawara. 2007. Weaving in stabled horses and its relationship to other behavioural traits. Appl Anim Behav Sci 106, 134-143.

Parker M, ES Redhead, D Goodwin, SD McBride. 2008. Impaired instrumental choice in crib biting horses (Equus caballus). Behav Brain Res 191, 137-140.

Phillips PEM, GD Stuber, MLAV Heien, RM Wightman, RM Carelli. 2003. Subsecond dopamine release promotes cocaine seeking. Nature 422 , 614-618.

Redbo I, P Redbo-Torstensson, FO Odberg, A Hedendahl, J Holm. 1998. Factors affecting behavioural disturbances in race horses. Anim Sci 66, 475-481.

Robbins TW. 1976. Relationship between reward-enhancing and stereotypical effects of psychomotor stimulant drugs. Nature 264, 57-59.

Rowe JB, DW Pethick, MJ Lees. 1994. Prevention of acidosis and laminitis associated with grain feeding in horses. J Nutr 124, 2742-2744.

Tinbergen N. 1951. The study of instinct. Oxford university press, New York, USA.

Vecchiotti GG, R Galanti. 1986. Evidence of heredity of cribbing, weaving and stall walking in thoroughbred horses. Livest Prod Sci 14, 91-95.

Waring GH. 2003. Ingestive behavior. Chapter 9. In: Horse behavior. William Andrew publishing, New Cork, USA, Pp 124-143.

Waters AJ, CJ Nicol, NP French. 2002. Factors influencing the development of stereotypic and redirected behaviours in young horses: findings of a four year prospective epidemiological study. Equine Vet $J 34,572-579$.

Wiepkema PR, DM Broom, I Duncan, G van Putten. 1983. Abnormal behaviour in farm animals - Report of the commission of the European communities. Commission of the European Communities, Brussels, Belgium.

Wiepkema PR, JM Koolhaas. 1993. Stress and animal welfare. Anim Welf 2, 195-218

Winskill LC, NK Waran, RJ Young. 1996. The effect of a foraging device (a modified Edinburgh foodball) on the behaviour of the stabled horse. Appl Anim Behav Sci 48, 25-35. 